\title{
miR-486-5p is upregulated in osteoarthritis and inhibits chondrocyte proliferation and migration by suppressing SMAD2
}

\author{
JUNKAI SHI $^{1 *}, \mathrm{KANSUO} \mathrm{GUO}^{1 *}, \mathrm{SHILE} \mathrm{SU}^{2}, \mathrm{JUN} \mathrm{LI}^{1}$ and CHUNHUI LI ${ }^{1}$ \\ ${ }^{1}$ Department of Orthopedics, Lianyungang Oriental Hospital; ${ }^{2}$ Department of Orthopedics, \\ Chinese People's Liberation Army No. 149 Hospital, Lianyungang, Jiangsu 222042, P.R. China
}

Received April 19, 2017; Accepted September 28, 2017

DOI: $10.3892 / \mathrm{mmr} .2018 .8931$

\begin{abstract}
Osteoarthritis (OA) is the most common form of arthritis and is caused by the breakdown of joint cartilage. The present study aimed to investigate an effective method for the treatment of OA. It was demonstrated that, compared with other patients, patients with OA exhibited lower mRNA expression levels of SMAD family member 2 (SMAD2). MicroRNA (miR)-486-5p was predicted to bind with SMAD2, which was verified by dual-luciferase reporter assay. Compared withcontrol patients who had no known history of OA or rheumatoid arthritis, patients with OA exhibited higher miR-486-5p expression level. Treatment with miR-486-5p mimics inhibited proliferation and migration of CHON-001 human chondrocytes, and also inhibited the expression levels of type II collagen and aggrecan. However, treatment with a miR-486-5p inhibitor promoted proliferation and migration, and the expression of type II collagen and aggrecan. Short interfering RNA-directed silencing of SMAD2 reversed the upregulated proliferation and migration and the expression level of type II collagen and aggrecan induced by the miR-486-5p inhibitor. In conclusion, the results of the present study indicated that miR-486-5p was upregulated in OA and may inhibit chondrocyte proliferation and migration by suppressing SMAD2.
\end{abstract}

\section{Introduction}

Osteoarthritis (OA) is a disease of the joints; it is the most common form of arthritis and is caused by the breakdown of joint cartilage and underlying bone (1). In 2004, OA led to moderate-severe disability in 43.4 million people

Correspondence to: Dr Shile Su, Department of Orthopedics, Chinese People's Liberation Army No. 149 Hospital, 127 Haitang Road, Lianyungang, Jiangsu 222042, P.R. China

E-mail: sushile0326@163.com

*Contributed equally

Key words: osteoarthritis, microRNA-486-5p, SMAD family member 2, cell proliferation, migration worldwide (2). In 2010, 3.8\% of the global population were affected by OA $(3,4)$.

OA affects work and normal daily activities, with joint pain and stiffness being the most frequent symptoms. Abnormal development of a joint or limb, inherited factors and previous joint injury may result in OA, and the risk of developing OA is greater in people who are overweight, have different leg lengths or have jobs with high joint stress $(5,6)$. OA develops as cartilage is lost and the underlying bone is affected (5). Balanced water content in healthy cartilage is maintained by the compressive force, which drives water out, and hydrostatic/osmotic pressures that draw water in $(7,8)$. Collagen fibres exhibit a compressive force pushing water out which is balanced by the Gibbs-Donnan effect and by cartilage proteoglycans that generate osmotic pressure to draw water in (8). However, during the progression of OA, the collagen matrix is disorganized and the breakdown of collagen fibers lead to an overall increase in water content (9).

SMAD family member 2 (SMAD2) is a protein that serves a crucial role in transmitting the extracellular signals from ligands of the transforming growth factor $\beta$ (TGF- $\beta$ ) superfamily into cell nucleus, which leads to the modulation of a number of cellular processes, including cell proliferation, apoptosis and differentiation $(10,11)$. In an OA mouse model, SMAD2 was demonstrated to be hyperphosphorylated, which implied that its activation may be connected to the development of OA (12). SMAD2 expression was also reported to be decreased in OA tissues (13).

MicroRNAs (miRNAs) are small non-coding RNA molecules that function in the post-transcriptional regulation of gene expression $(14,15)$. It is unknown whether there are miRNAs that could target SMAD2 and serve a role in the treatment of OA.

The present study aimed to investigate the expression and role miR-486-5p in osteoarthritis, and demonstrated that miR-486-5p was upregulated in OA. Furthermore, miR-486-5p may inhibit chondrocyte proliferation and migration by targeting SMAD2, indicating the crucial role of miR-486-5p in OA. Therefore, miR-486-5p maybe a potential target in the treatment of OA.

\section{Materials and methods}

Patients. Articular cartilage tissues from $25 \mathrm{OA}$ patients undergoing total knee replacement surgery (male, 14; female, 11; 
age $62.4 \pm 6.2$ years) were considered to be the experimental group and knee articular cartilage tissues from 25 non-OA patients (male, 16; female, 11; age 60.1 \pm 7.2 years) with femoral neck fracture who had no known history of OA or rheumatoid arthritis served as the control. All samples were collected at the Chinese People's Liberation Army No. 149 Hospital from March 2015 to August 2016. The present study was approved by the Ethics committee of Lianyungang Oriental Hospital (Lianyungang, China). All of the participants in the present study provided written informed consent to the ethics committees of the Chinese People's Liberation Army No. 149 Hospital (Lianyungang, China).

Cell culture. CHON-001 human chondrocyte cells were cultured in Dulbecco's modified Eagle's medium/nutrient mixture F12 (DMEM/F12; Gibco; Thermo Fisher Scientific, Inc., Waltham, MA, USA), supplemented with antibiotic and antimycotic agents $(10,000$ units $/ \mathrm{ml}$ penicillin, 10,000 $\mu \mathrm{g} / \mathrm{ml}$ streptomycin and $25 \mu \mathrm{g} / \mathrm{ml}$ Fungizone; Gibco; Thermo Fisher Scientific, Inc.) in standard culture conditions $\left(37^{\circ} \mathrm{C}, 5 \% \mathrm{CO}_{2}\right.$ and $95 \%$ humidity).

Cell transfection. miR-486-5p mimics (50 nM; cat. no. miR10003130-1-5), miR-486-5p inhibitor (100 nM; cat. no. miR20003130-1-5), the negative control of miR-486-5p (NC, 50 nM; cat no. miR01101-1-5), control siRNA (si-Control), and SMAD2 siRNA were purchased from Guangzhou RiboBio Co., Ltd. (Guangzhou, China). To perform CHON-001 cell transfection, Lipofectamine ${ }^{\circledR} 2000$ (Invitrogen; Thermo Fisher Scientific, Inc.) was used according to the manufacturer's protocol. Transfection efficiency was determined using reverse transcription-quantitative polymerase chain reaction (RT-qPCR) as described below. The cells were then incubated for $24 \mathrm{~h}$ at $37^{\circ} \mathrm{C}$ and $5 \% \mathrm{CO}_{2}$ until further analysis.

MTT assay. CHON-001 cells $\left(5 \times 10^{3}\right.$ cells/well) were grown in 96-well plates. A total of $24 \mathrm{~h}$ following transfection with either miR-486-5p mimics, miR-486-5p inhibitor or NC at $37^{\circ} \mathrm{C}$. Subsequently, MTT solution $(20 \mu \mathrm{l})$ was added into each well at 24, 48 and $72 \mathrm{~h}$, and the plates were incubated at $37^{\circ} \mathrm{C}$ for an additional $4 \mathrm{~h}$. The MTT solution was aspirated and dimethyl sulfoxide $(150 \mu \mathrm{l})$ was added prior to assessment of absorbance at A570 nm; the value represents the cell proliferation of each well examined.

Transwell migration assay. The migratory abilities of CHON-001 cells were tested using 24-well Transwell chambers, with upper and lower culture compartments separated by polycarbonate membranes. Cells $\left(2 \times 10^{4}\right)$ were suspended in $100 \mu \mathrm{l}$ serum-free DMEM and seeded into the top chambers. The bottom chambers contained $0.5 \mathrm{ml}$ DMEM with $10 \%$ fetal bovine serum (FBS, Thermo Fisher Scientific, Inc.). Following sub-culturing for $\sim 24 \mathrm{~h}$, cells on the upper surface of membrane were removed by cotton tips. Cells that migrated to the lower surface were fixed with $10 \%$ formalin for $30 \mathrm{~min}$ at room temperature. Migratory abilities were evaluated by counting the number of cells stained with hematoxylin and eosin for $15 \mathrm{~min}$ at $37^{\circ} \mathrm{C}$ cells using an inverted microscope (magnification, x200; Olympus Corporation, Japan).
Dual-luciferase assay. TargetScan bioinformatics software (TargetScanHuman 7.1; www.targetscan.org/vert_71) was used to predict the target genes of miR-486-5p, which indicated that SMAD2 is potential target of miR-486-5p. To verify that miR-486-5p directly targets the 3'-untranslated region (UTR) of SMAD2, the vectors SMAD2-3'UTR-wild type (WT, GAGCTCTCCCAAAGGTTTATTAATAACAGTAGT AGTTATGTGTACAGGTAATGTATCATCTCGAG) and SMAD2-3'UTR-mutant (MUT, GAGCTCTCCCAAAGGTTT ATTAATAACAGTAGTAGTTATGTCATGTCCTAATGTATC ATCTCGAG) of pMIR-REPORT (GeneCopoeia, Inc., Rockville, MD, USA), which respectively contained the wild-type and mutated 3'UTR of SMAD2 mRNA, were established. For the luciferase reporter assay, CHON-001 cells $\left(5 \times 10^{4}\right.$ cells/well) were seeded into 24 -well plates. CHON-001 cells were co-transfected with either SMAD2-3'UTR-WT or SMAD2-3'UTR-MUT and either miR-486-5p mimics or miR-negative control using Lipofectamine ${ }^{\circledR} 2000$ (Invitrogen; Thermo Fisher Scientific, Inc.) at $37^{\circ} \mathrm{C}$ for $48 \mathrm{~h}$. Then, cells were collected and the luciferase activity was analyzed using the Dual-Luciferase Reporter Assay system (Promega Corporation, Madison, WI, USA) according to the manufacturer's protocols. Renilla luciferase activity was normalized to firefly luciferase activity.

Total RNA isolation and RT-qPCR. Micro-dismembrator was applied to homogenize deep-frozen articular cartilage tissues at $4^{\circ} \mathrm{C}$ for $1 \mathrm{~min}$ at a speed of $1,500 \mathrm{RPM}$. Total RNA was isolated using an RNeasy Mini kit (Qiagen, Inc., Valencia, CA, USA) according to manufacturer's protocol. cDNA was synthesized by reverse transcription using the TaqMan MicroRNA Reverse Transcription kit (Applied Biosystems; Thermo Fisher Scientific, Inc.) and was used in the qPCR reactions according to the manufacturer's protocol. Amplification conditions for qPCR were as follows: $95^{\circ} \mathrm{C}$ for $5 \mathrm{~min}$, followed by 40 cycles of denaturation at $95^{\circ} \mathrm{C}$ for $15 \mathrm{sec}$ and annealing/elongation at $60^{\circ} \mathrm{C}$ for $30 \mathrm{sec}$. GAPDH was used as a reference gene. The $2^{-\Delta \Delta \mathrm{Cq}}$ method was used to calculate the relative quantities of each gene (16). Primer sequences are listed in Table I.

Western blot analysis. Logarithmic growth phase cells (90\% confluence) were harvested and lysed by radioimmunoprecipitation assay lysis buffer (Beyotime Institute of Biotechnology, Haimen, China). The concentration of protein was detected by BCA assay (Thermo Fisher Scientific, Inc.) per as the manufacturer's protocol. Protein lysates (25 $\mu \mathrm{g}$ per lane) were separated by $12 \%$ SDS-PAGE, transferred to polyvinylidene difluoride (PVDF) membranes. Following blocking with 5\% skimmed milk for $2 \mathrm{~h}$ at room temperature, the membranes were incubated with primary antibodies against SMAD2 (1:1,000; cat. no. 5339; Cell Signaling Technology, Inc., Danvers, MA, USA), type II collagen (1:1,000; cat. no. ab34712), aggrecan (1:1,000; cat. no. ab36861; both Abcam, Cambridge, UK) and $\beta$-actin (1:2,000; cat. no. 4970; Cell Signaling Technology, Inc.) at $4^{\circ} \mathrm{C}$ overnight. Subsequently, the PVDF membranes were washed 3 times with TBS containing 0.1\% Tween-20 and incubated with Anti-rabbit IgG, HRP-linked Antibody (1:5,000; cat no. 7074; Cell Signaling Technology, Inc.) at room temperature for $\sim 1 \mathrm{~h}$. Blots were developed with SuperSignal West Femto Maximum 
Table I. Primer sequences for reverse transcription-qPCR.

\begin{tabular}{ll}
\hline Gene & \multicolumn{1}{c}{ Primer sequence $\left(5^{\prime} \rightarrow 3^{\prime}\right)$} \\
\hline SMAD2 & F: ATGTCGTCCATCTTGCCATTC \\
Type II collagen & F: AACCGTCCTGTTTCTTTAGCTT \\
& R: CACTGAGTGGAAGAGTGGAG \\
Aggrecan & F: CTAGAGATCAGTGGACTGCCT \\
miR-486-5p & R: TCTGGAGCTGTGCAGTCTAGTGG \\
& F: CTCGCTTCGGCAGCACA \\
U6 & F: ACGCTTCACGAATTTGCGT \\
& F: Universal_RNU6B_Primer \\
GAPDH & R: Uni-miR qPCR Primer \\
& F: CTTTGGTATCGTGGAAGGACTC \\
& R: GTAGAGGCAGGGATGATGTTCT
\end{tabular}

$\mathrm{F}$, forward; miR, microRNA; qPCR, quantitative polymerase chain reaction; R, reverse; SMAD2, SMAD family member 2; Uni, universal.

Sensitivity Substrate (Pierce; Thermo Fisher Scientific, Inc.), and the ChemiDoc XRS+ system (Bio-Rad Laboratories, Inc., Hercules, CA, USA) was used for protein band observation according to the manufacturer's protocols. Data were analyzed by densitometry using Image Pro Plus v.6.0 software (Media Cybernetics, Inc., Rockville, MD, USA). $\beta$-actin served as an internal control for all experiments.

Statistical analysis. All the experiments were performed at least three times. Data are presented as the mean \pm standard deviation. One-way analysis of variance with Tukey's post hoc test was used to evaluate the effects of different treatments. Statistical analysis was performed with SPSS software version 20.0 (IBM Corp., Armonk, NY, USA). P<0.05 was considered to indicate a statistically significant difference.

\section{Results}

Patients with OA exhibit lower SMAD2 mRNA expression and higher miR-486-5p expression. mRNA expression levels of SMAD2 were examined by RT-qPCR in the articular cartilage tissues of patients with and without OA. The results demonstrated that SMAD2 expression was significantly decreased in patients with OA compared with the control patients without OA (P<0.01; Fig. 1A). The expression levels of miR-486-5p in the two articular cartilage tissues were also examined by RT-qPCR, and the results demonstrated a significantly increased expression level of miR-486-5p in patients with OA compared with the control patients ( $\mathrm{P}<0.01$; Fig. 1B).

SMAD2 is a direct target of miR-486-5p. TargetScan was used to predict miRNAs that may target SMAD2 mRNA, and miR-486-5p exhibited a relatively high score and was indicated to bind to the 3'-UTR of SMAD2 (Fig. 2A). SMAD2 was subsequently confirmed as a direct target of miRNA-486-5p by dual-luciferase reporter assay, which demonstrated that the relative luciferase activity was significantly decreased in
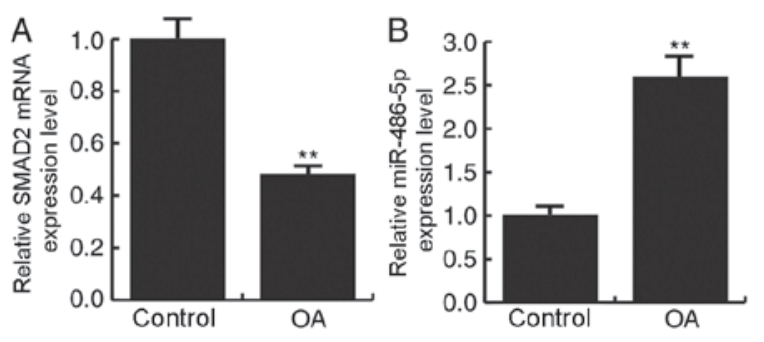

Figure 1. Relative expression levels of SMAD2 mRNA and miR-486-5p in patients with OA. Relative expression of SMAD2 and miR-486-5p in patients with and without $\mathrm{OA}$ were determined by reverse transcription-quantitative polymerase chain reaction. (A) Relative mRNA expression level of SMAD2. (B) Relative expression level of miR-486-5p. ${ }^{* *} \mathrm{P}<0.01$ vs. Control. miR, microRNA; OA, osteoarthritis; SMAD2, SMAD family member 2.

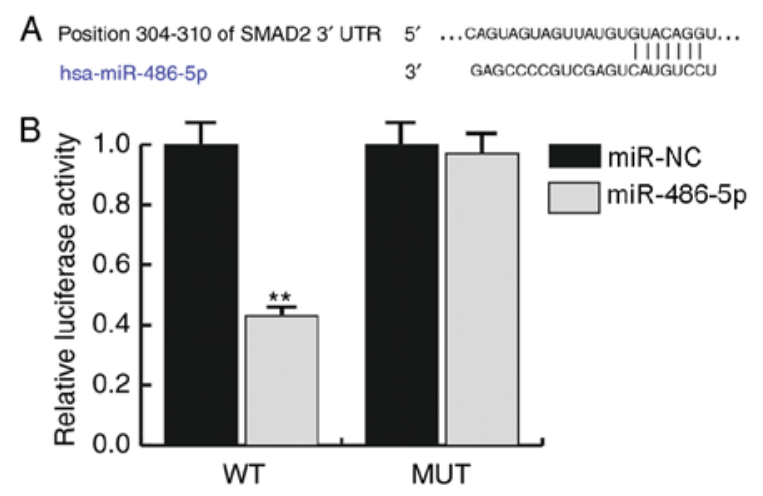

Figure 2. SMAD2 is a direct target of miR-486-5p. (A) TargetScan was used to predict the interactions between miR-486-5p and the wild-type 3'UTR of SMAD2. (B) Luciferase activity was detected by dual-luciferase assay. Data are presented as the mean \pm standard deviation; ${ }^{* *} \mathrm{P}<0.01$ vs. miR-NC. SMAD2, SMAD family member 2; miR, microRNA; MUT, mutant; NC, negative control; UTR, untranslated region; WT, wild-type.

the SMAD2-3'UTR-WT group transfected with miR-486-5p mimics compared with those cells co-transfected with SMAD2-3'UTR-WT and miR-NC (P<0.01; Fig. 2B).

miR-486-5p mimics inhibit $\mathrm{CHON-001} \mathrm{cell} \mathrm{proliferation} \mathrm{and}$ migration and the expression of type II collagen and aggrecan. To investigate the role of miR-486-5p in the development of OA, CHON-001 cells were transfected with miR-486-5p mimics or miR-486-5p inhibitor. miR-486-5p expression was significantly enhanced by treatment with miR-486-5p mimics and significantly inhibited by miR-486-5p inhibitor treatment $(\mathrm{P}<0.05$ vs. NC; Fig. 3A). As expected, the miR- $486-5 \mathrm{p}$ mimics significantly suppressed the expression level of SMAD2 mRNA ( $\mathrm{P}<0.05$; Fig. 3B) and notably reduced SMAD2 protein expression (Fig. 3C and D) compared with NC group. Conversely, treatment with the miR-486-5p inhibitor resulted in the opposite effects on SMAD2 mRNA and protein expression levels ( $\mathrm{P}<0.05$; Fig. 3B-D).

The effects of miR-486-5p on CHON-001 cell proliferation and migration were determined by MTT and Transwell migration assays, respectively. Compared with the miR-NC transfected cells, the proliferative ability of cells transfected with miR-486-5p mimics was significantly inhibited $(\mathrm{P}<0.05$; Fig. $4 \mathrm{~A})$. In addition, number of migrated cells in the miR-486-5p mimics transfected group was decreased 
A

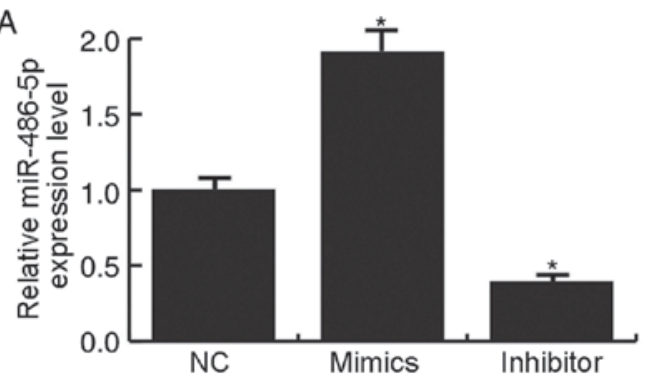

C

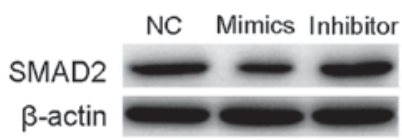

$B$
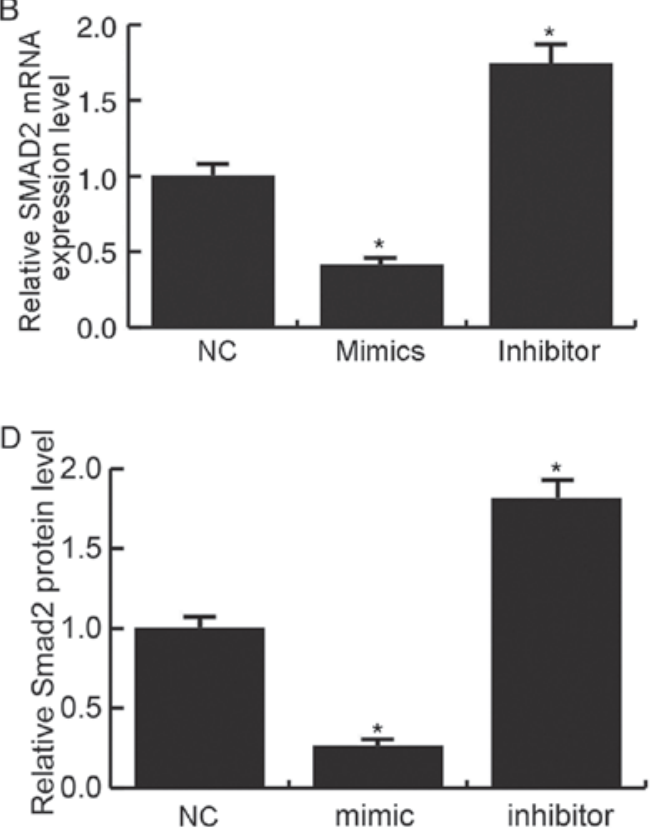

Figure 3. Expression levels of miR-486-5p and SMAD2 in CHON-001 cells in different treatment groups. Following transfection with miR-486-5p mimics or inhibitor, the expression level of miR-486-5p and SMAD2 in CHON-001 was detected by reverse transcription-quantitative polymerase chain reaction and western blotting. (A) Relative expression levels of miR-486-5p. (B) Relative expression levels of SMAD2 mRNA. (C and D) Protein expression levels of SMAD2. Data are presented as the mean \pm standard deviation; ${ }^{*} \mathrm{P}<0.05$ vs. NC. miR, microRNA; SMAD2, SMAD family member 2.

compared with the miR-NC group, which indicated lower migratory ability following transfection with miR-486-5p mimics (Fig. 4B).

The expression of type II collagen and aggrecan are important for the development of articular cartilage tissue. RT-qPCR results demonstrated that the mRNA expression levels of type II collagen and aggrecan were significantly decreased in response to $\mathrm{miR}-486-5 \mathrm{p}$ mimics transfection compared with cells in the miR-NC transfected group $(\mathrm{P}<0.01$; Fig. 5A). Results from western blotting also indicated that miR-486-5p mimics transfection notably reduced the protein expression levels of type II collagen and aggrecan (Fig. 5B-D).

miR-486-5p inhibitor promotes $\mathrm{CHON-001} \mathrm{cell} \mathrm{proliferation}$ and migration and the expression of type II collagen and aggrecan. To further confirm the role of miR-486-5p in OA development, a miR-486-5p inhibitor was transfected into CHON-001 cells. Compared with the NC, cells transfected with miR-486-5p inhibitor exhibited increased proliferative and migratory capability (Fig. 4A and B, respectively). In addition, the mRNA expression levels of type II collagen and aggrecan were significantly increased in response to miR-486-5p inhibitor treatment $(\mathrm{P}<0.01$; Fig. 5A). Furthermore, the protein expression levels of type II collagen and aggrecan significantly increased following miR-486-5p inhibitor treatment $(\mathrm{P}<0.01$; Fig. 5B-D).

Silencing of SMAD2 reverses the miR-486-5p inhibitor-induced upregulation of $\mathrm{CHON}-001$ cell proliferation and migration, and the expression levels of type II collagen and aggrecan. SMAD2 serves a key role in OA development. To determine if miRNA-486-5p affected chondrocytes through SMAD2, Small interfering (si)RNAs were used to
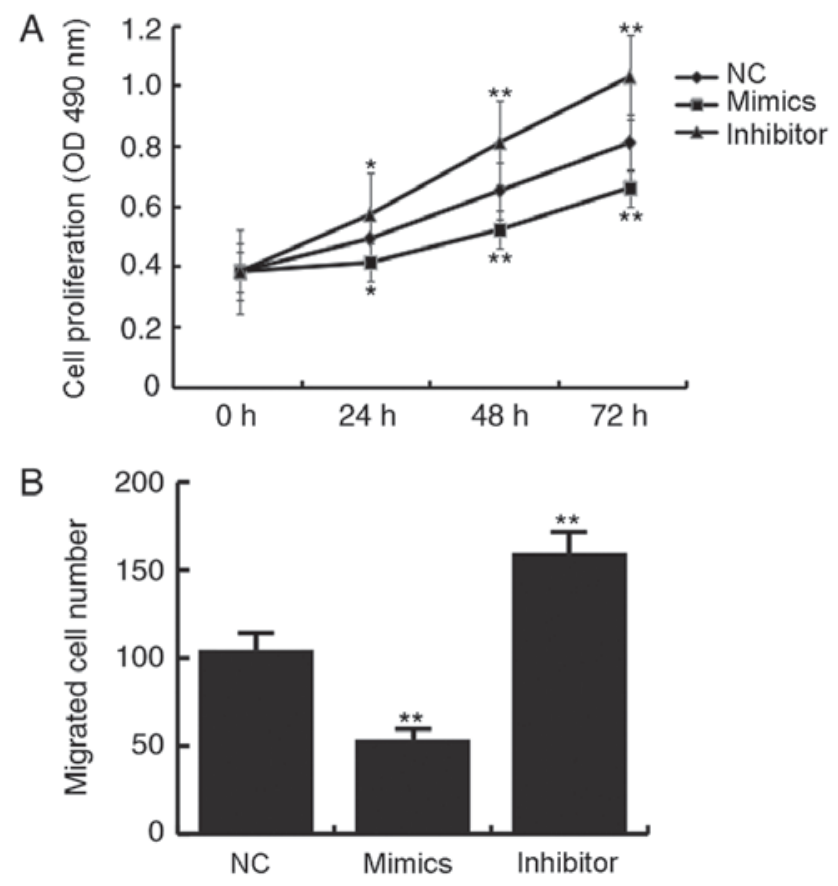

Figure 4. Effects of miR-486-5p on CHON-001 cell proliferation and migration. A total of $24 \mathrm{~h}$ following cell transfection, $\mathrm{CHON}-001$ cell proliferation and migration were determined by MTT and Transwell migration assays, respectively. (A) Effects of miR-486-5p overexpression and inhibition on CHON-001 cell proliferation. (B) Effects of miR-486-5p overexpression and inhibition on $\mathrm{CHON}-001$ cell migration. Data are presented as the mean \pm standard deviation; ${ }^{*} \mathrm{P}<0.05$ and ${ }^{* *} \mathrm{P}<0.01$ vs. NC. miR, microRNA; $\mathrm{NC}$, negative control; OD, optical density.

silence SMAD2 expression in CHON-001 cells. Western blotting demonstrated that SMAD2 protein expression level 

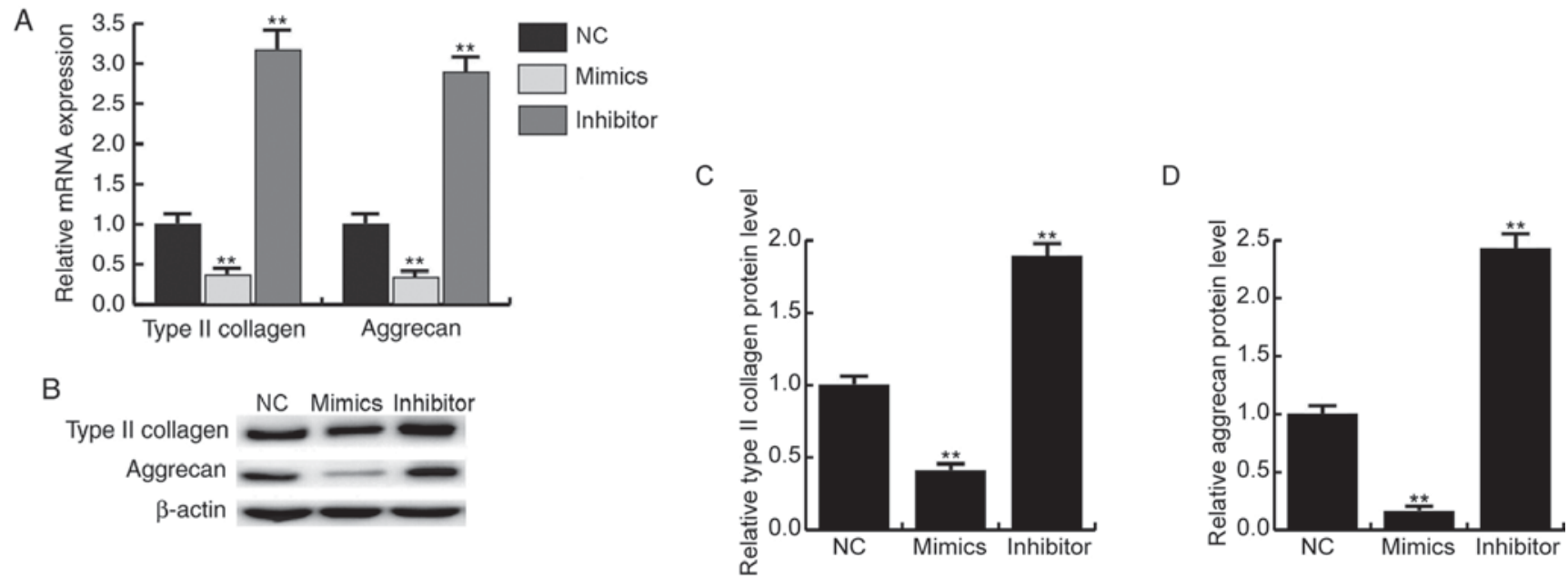

Figure 5. Effects of microRNA-486-5p on type II collagen and aggrecan expression. A total of $24 \mathrm{~h}$ following cell transfection, the expression mRNA and protein expression levels of type II collagen and aggrecan were detected by reverse transcription-quantitative polymerase chain reaction and western blotting, respectively. (A) Relative mRNA expression levels of type II collagen and aggrecan. Data are presented as the mean \pm standard deviation; ${ }^{* *} \mathrm{P}<0.01$ vs. NC. (B-D) Protein expression levels of type II collagen and aggrecan. NC, negative control.
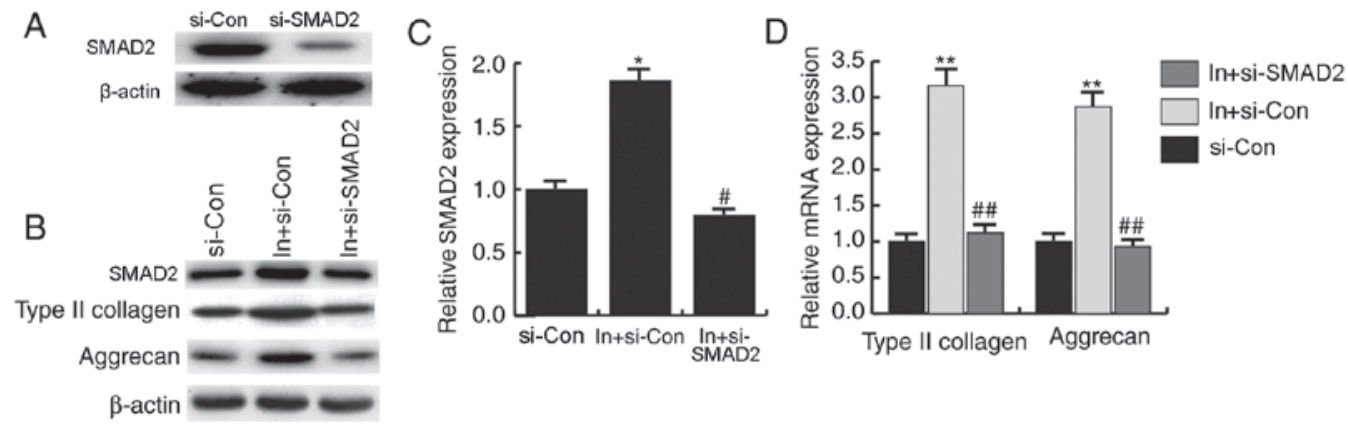

Figure 6. Effects of siRNA-SMAD2 on SMAD2, type II collagen and aggrecan expression. (A) SMAD2 protein expression is reduced in cells treated with si-SMAD2. (B) The protein expression levels of SMAD2, type II collagen and aggrecan in different groups. (C) SMAD2, (D) type II collagen and aggrecan mRNA expression levels in the different groups. Data are presented as the mean \pm standard deviation; ${ }^{*} \mathrm{P}<0.05$ and ${ }^{* *} \mathrm{P}<0.01$ vs. si-Con; ${ }^{\#} \mathrm{P}<0.05$ and ${ }^{\# \#} \mathrm{P}<0.01$ vs. In + si-Con. Con, control; in, miRNA-486-5p inhibitor; si, small interfering; SMAD2, SMAD family member 2.
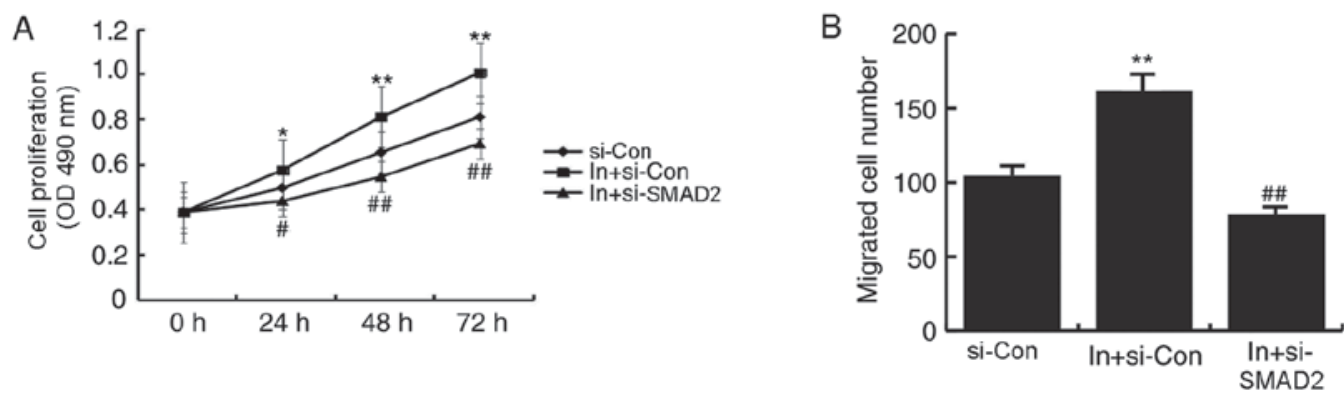

Figure 7. Effects of siRNA-SMAD2 on CHON-001 cell proliferation and migration. Effects of siRNA-SMAD2 on proliferation and migration were determined by MTT and Transwell migration assay, respectively. (A) Effects of siRNA-SMAD2 on CHON-001 cell proliferation. (B) Effects of siRNA-SMAD2 on CHON-001 cell migration. Data are presented as the mean \pm standard deviation. ${ }^{*} \mathrm{P}<0.05$ and ${ }^{* * *} \mathrm{P}<0.01$ vs. si-Con; ${ }^{*} \mathrm{P}<0.05$ and ${ }^{\# \#} \mathrm{P}<0.01$ vs. In+si-Con. si-Con, control siRNA; In, miRNA-486-5p inhibitor; si, small interfering; SMAD2, SMAD family member 2.

was reduced by SMAD2 siRNA transfection compared with si-Control-treated cells (Fig. 6A). Western blotting and RT-qPCR results demonstrated that, compared with cells transfected with miRNA-486-5p inhibitor + si-Con, SMAD2 protein and mRNA expression levels were decreased in cells transfected with miRNA-486-5p inhibitor + si-SMAD2 $(\mathrm{P}<0.05$; Fig. $6 \mathrm{~B}$ and $\mathrm{C})$. In addition, the miRNA-486-5p inhibitor-induced increase of type II collagen and aggrecan expression levels were reversed by si-SMAD2 co-transfection (Fig. 6B and D). Notably, the miR-486-5p inhibitor-induced increase in proliferation and migration was inhibited by SMAD2 silencing (Fig. 7A and B, respectively). These results indicated that silencing of SMAD2 reversed the upregulation of CHON-001 cell proliferation and migration and the level of 
type II collagen and aggrecan expression that were induced by miR-486-5p inhibitor treatment.

\section{Discussion}

$\mathrm{OA}$ is the most prevalent chronic joint disease that affects the majority of people over 65 years old (17). The pathogenesis of $\mathrm{OA}$ is very complex and involves several molecular mechanisms, such as disregulation of the TGF- $\beta$ signaling pathway and the Wnt $/ \beta$-catenin signaling pathway $(18,19)$. miRNAs were previously indicated as contributing to the disrupted signaling network in OA (20), and a number of specific miRNAs have been identified to be crucial during OA pathogenesis (21). As a result, the discovery of key miRNAs that may serve a role in the development of OA may provide valuable insight for designing effective miRNA-targeted therapies. In the present study, it was suggested that miR-486-5p may be involved in development of OA and therefore may be a promising target for the treatment of OA.

Loss of control of TGF- $\beta$ signaling is frequently observed in OA $(22,23)$. A previous study indicated that TGF- $\beta$ signaling contributed to the development of OA through the activin receptor-like kinase (ALK) 5/SMAD2/SMAD3 pathway or the ALK1/SMAD2/SMAD5 pathway (24). SMAD2 appeared to exhibit a stronger effect on chondrogenesis compared with SMAD3 in vivo (25). In addition, a lower level of SMAD2 protein expression was detected in patients with OA compared with in patients without OA (13). TGF- $\beta$ signaling may activate the mitogen-activated protein kinase 1/extracellular signal-regulated kinase signaling pathway and the SMAD2/SMAD3 signaling pathway to induce the expression of type II collagen and aggrecan in rat chondrocytes (26). In human chondrocyte cell line $\mathrm{CHON}-001$, silencing of SMAD2 expression was demonstrated to trigger a decrease in type II collagen and aggrecan protein levels, which suggested a crucial role for SMAD2 in OA development.

In addition to being regulated by TGF- $\beta$ signaling, SMAD2 is also controlled by several other molecules, including miRNAs. For example, miR-155 was reported to directly target SMAD2 and repress SMAD2 expression (27). miR-486-5p regulates multiple oncogenes and was demonstrated to be closely linked to the development of cancer $(28,29)$. A previous study indicated that miR-486-5p may regulate SMAD2 expression in lens epithelial cells (30). However, the role of SMAD2 in OA remains elusive. In the present study, it was demonstrated that SMAD2 expression was decreased and that miR-486-5p expression was elevated in patients with OA. The findings of the present study were consistent with previous research $(13,31)$. Additionally, Liu et al (30) reported that miR-486-5p may regulate SMAD2 expression in lens epithelial cells. The present study investigated the association between miR-486-5p and SMAD2 in CHON-001 cells and the results indicated that SMAD2 was demonstrated to be a direct target of miR-486-5p. Furthermore, the effect of miR-486-5p on chondrocyte were also investigated in the present study. The findings suggested that overexpression of miR-486-5p decreased chondrocyte proliferation and migration. Conversely, inhibition of miR-486-5p resulted in increased chondrocyte proliferation and migration. Notably, this increase in proliferation and migration was reversed by SMAD2 silencing. The expression levels of type II collagen and aggrecan were also decreased in response to the miR-486-5p mimics transfection, which may be enhanced by miR-486-5p inhibitor. SMAD2 silencing reversed the miR-486-5p inhibitor-induced upregulated expression level of type II collagen and aggrecan.

In conclusion, the present study demonstrated that miR-486-5p targeted SMAD2 mRNA, a key gene in OA development. By targeting SMAD2, miR-486-5p negatively regulated the expression levels of type II collagen and aggrecan in chondrocytes. Therefore, the loss of miR-486-5p may increase proliferation and migration of chondrocytes. The results of the present study suggested a crucial role for miR-486-5p in OA, which indicated that miR-486-5p may represent a potential marker and target for the diagnosis and treatment of OA.

\section{Acknowledgements}

Not applicable.

\section{Funding}

No funding was received.

\section{Availability of data and materials}

The analyzed data sets generated during the present study are available from the corresponding author on reasonable request.

\section{Authors' contributions}

JS, SS and KG made substantial contributions to the concept and design of the study, literature search and manuscript preparation. JL and CL made substantial contributions to statistical analysis and data interpretation.

\section{Ethics approval and consent to participate}

The present study was approved by the Ethics committee of Lianyungang Oriental Hospital (Lianyungang, China). Written informed consent was obtained from all patients.

\section{Consent for publication}

Not applicable.

\section{Competing interests}

The authors declare that they have no competing interests.

\section{References}

1. Arden N, Blanco F, Cooper C, Guermazi A, Hayashi D, Hunter D, Javaid MK, Rannou F, Roemer FW and Reginster JY: Atlas of osteosteoarthritis. Springer Healthcare Ltd: 69-82, 2014.

2. World Health Organization: The Global Burden of Disease. 2004 Update. World Health Organization, Geneva, 2008.

3. Cross M, Smith E, Hoy D, Nolte S, Ackerman I, Fransen M, Bridgett L, Williams S, Guillemin F, Hill CL, et al: The global burden of hip and knee osteoarthritis: Estimates from the global burden of disease 2010 study. Ann Rheum Dis 73: 1323-1330, 2014. 
4. March L, Smith EU, Hoy DG, Cross MJ, Sanchez-Riera L, Blyth F, Buchbinder R, Vos T and Woolf AD: Burden of disability due to musculoskeletal (MSK) disorders. Best Pract Res Clin Rheumatol 28: 353-366, 2014.

5. Chu CR, Millis MB and Olson SA. Osteoarthritis: From Palliation to Prevention: AOA Critical Issues. J Bone Joint Surg Am 96: e130, 2014.

6. Glyn-Jones S, Palmer AJ, Agricola R, Price AJ, Vincent TL, Weinans H and Carr AJ: Osteoarthritis. Lancet 386: 376-387, 2015

7. Sanchez-Adams J, Leddy HA, McNulty AL, O'Conor CJ and Guilak F: The mechanobiology of articular cartilage: Bearing the burden of osteoarthritis. Curr Rheumatol Rep 16: 451, 2014.

8. Maroudas AI: Balance between swelling pressure and collagen tension in normal and degenerate cartilage. Nature 260: 808-809, 1976.

9. Chou MC, Tsai PH, Huang GS, Lee HS, Lee $\mathrm{CH}$, Lin MH, Lin CY and Chung HW: Correlation between the MR T2 value at $4.7 \mathrm{~T}$ and relative water content in articular cartilage in experimental osteoarthritis induced by ACL transection. Osteoarthritis Cartilage 17: 441-447, 2009.

10. Eppert K, Scherer SW, Ozcelik H, Pirone R, Hoodless P, Kim H, Tsui LC, Bapat B, Gallinger S, Andrulis IL, et al: MADR2 maps to $18 \mathrm{q} 21$ and encodes a TGFbeta-regulated MAD-related protein that is functionally mutated in colorectal carcinoma. Cell 86 : 543-552, 1996.

11. Riggins GJ, Thiagalingam S, Rozenblum E, Weinstein CL, Kern SE, Hamilton SR, Willson JK, Markowitz SD, Kinzler KW and Vogelstein B: Mad-related genes in the human. Nat Genet 13: 347-349, 1996

12. Long E, Motwani R, Reece D, Pettit N, Hepworth J, Wong P, Reynolds P and Seegmiller R: The role of TGF- $\beta 1$ in osteoarthritis of the temporomandibular joint in two genetic mouse models. Arch Oral Biol 67: 68-73, 2016.

13. Madej W, Buma P and van der Kraan P: Inflammatory conditions partly impair the mechanically mediated activation of Smad2/3 signaling in articular cartilage. Arthritis Res Ther 18: 146, 2016.

14. Ambros V: The functions of animal microRNAs. Nature 431: 350-355, 2004.

15. Bartel DP: MicroRNAs: Genomics, biogenesis, mechanism, and function. Cell 116: 281-297, 2004.

16. Livak KJ and Schmittgen TD: Analysis of relative gene expression data using real-time quantitative PCR and the 2(-Delta Delta $\mathrm{C}(\mathrm{T})$ ) method. Methods 25: 402-408, 2001.

17. Dahaghin S, Bierma-Zeinstra SM, Ginai AZ, Pols HA, Hazes JM and Koes BW: Prevalence and pattern of radiographic hand osteoarthritis and association with pain and disability. Ann Rheum Dis 64: 682-687, 2005.

18. Blaney Davidson EN, Vitters EL, van der Kraan PM and van den Berg WB: Expression of transforming growth factor-beta (TGFbeta) and the TGFbeta signalling molecule SMAD-2P in spontaneous and instability-induced osteoarthritis: Role in cartilage degradation, chondrogenesis and osteophyte formation. Ann Rheum Dis 65: 1414-1421, 2006.
19. Min JL, Meulenbelt I, Riyazi N, Kloppenburg M, Houwing-Duistermaat JJ, Seymour AB, Pols HA, van Duijn CM and Slagboom PE: Association of the Frizzled-related protein gene with symptomatic osteoarthritis at multiple sites. Arthritis Rheum 52: 1077-1080, 2005.

20. Iliopoulos D, Malizos KN, Oikonomou P and Tsezou A: Integrative microRNA and proteomic approaches identify novel osteoarthritis genes and their collaborative metabolic and inflammatory networks. PLoS One 3: e3740, 2008.

21. Sumiyoshi K, Kubota S, Ohgawara T, Kawata K, Nishida T, Shimo T, Yamashiro T and Takigawa M: Identification of miR-1 as a micro RNA that supports late-stage differentiation of growth cartilage cells. Biochem Biophys Res Commun 402: 286-290, 2010.

22. Blaney Davidson EN, van der Kraan PM and van den Berg WB: TGF-beta and osteoarthritis. Osteoarthritis Cartilage 15: 597-604, 2007

23. Itayem R, Mengarelli-Widholm S, Hulth A and Reinholt FP Ultrastructural studies on the effect of transforming growth factor 1 on rat articular cartilage. Apmis 105: 221-218, 1997.

24. Finnson KW, Parker WL, Ten Dijke P, Thorikay M and Philip A: ALK1 opposes ALK5/Smad3 signaling and expression of extracellular matrix components in human chondrocytes. J Bone Miner Res 23: 896-906, 2008.

25. Wang W, Song B, Anbarchian T, Shirazyan A, Sadik JE and Lyons KM: Smad2 and Smad3 regulate chondrocyte proliferation and differentiation in the growth plate. PLoS Genet 12: e1006352, 2016.

26. Zhu Y, Tao H, Jin C, Liu Y, Lu X, Hu X and Wang X: Transforming growth factor- $\beta 1$ induces type II collagen and aggrecan expression via activation of extracellular signal-regulated kinase $1 / 2$ and Smad2/3 signaling pathways. Mol Med Rep 12: 5573-9, 2015

27. Louafi F, Martinez-Nunez RT and Sanchez-Elsner T: MicroRNA-155 targets SMAD2 and modulates the response of macrophages to transforming growth Factor-\{beta\}. J Biol Chem 285: 41328-41336, 2010.

28. Wang J, Tian X, Han R, Zhang X, Wang X, Shen H, Xue L, Liu Y, Yan X, Shen J, et al: Downregulation of miR-486-5p contributes to tumor progression and metastasis by targeting protumorigenic ARHGAP5 in lung cancer. Oncogene 33: 1181-1189, 2014

29. Pang W, Tian X, Bai F, Han R, Wang J, Shen H, Zhang X, Liu Y, Yan X, Jiang F and Xing L: Pim-1 kinase is a target of miR-486-5p and eukaryotic translation initiation factor 4E, and plays a critical role in lung cancer. Mol Cancer 13: 240, 2014

30. Liu B, Sun J, Lei X, Zhu Z, Pei C and Qin L: MicroRNA-486-5p suppresses TGF- $\beta 2$-induced proliferation, invasion and epithelial-mesenchymal transition of lens epithelial cells by targetingSmad2. J Biosci 42: 575-584, 2017.

31. Kong R, Gao J, Si Y and Zhao D: Combination of circulating miR-19b-3p, miR-122-5p and miR-486-5p expressions correlates with risk and disease severity of knee osteoarthritis. Am J Transl Res 9: 2852-2864, 2017. 